\title{
Synaptic and Vesicular Coexistence of VGLUT and VGAT in Selected Excitatory and Inhibitory Synapses
}

\author{
Johannes-Friedrich Zander, ${ }^{1}$ Agnieszka Münster-Wandowski, ${ }^{1}$ Irene Brunk, ${ }^{1}$ Ingrid Pahner, ${ }^{1}$ Gisela Gómez-Lira, ${ }^{3}$ \\ Uwe Heinemann, ${ }^{2}$ Rafael Gutiérrez, ${ }^{3}$ Gregor Laube, ${ }^{1}$ and Gudrun Ahnert-Hilger ${ }^{1}$ \\ ${ }^{1}$ Institut für Integrative Neuroanatomie and ${ }^{2}$ Institut für Neurophysiologie and NeuroCure Research Center, Charité Centrum 2 für Grundlagenmedizin, \\ 10115 Berlin, Germany, and ${ }^{3}$ Departamento de Fisiología, Biofísica, y Neurociencias, Centro de Investigación y Estudios Avanzados del Instituto Politécnico \\ Nacional, México DF 0700, México
}

The segregation between vesicular glutamate and GABA storage and release forms the molecular foundation between excitatory and inhibitory neurons and guarantees the precise function of neuronal networks. Using immunoisolation of synaptic vesicles, we now show that VGLUT2 and VGAT, and also VGLUT1 and VGLUT2, coexist in a sizeable pool of vesicles. VGAT immunoisolates transport glutamate in addition to GABA. Furthermore, VGLUT activity enhances uptake of GABA and monoamines. Postembedding immunogold double labeling revealed that VGLUT1, VGLUT2, and VGAT coexist in mossy fiber terminals of the hippocampal CA3 area. Similarly, cerebellar mossy fiber terminals harbor VGLUT1, VGLUT2, and VGAT, while parallel and climbing fiber terminals exclusively contain VGLUT1 or VGLUT2, respectively. VGLUT2 was also observed in cerebellar GABAergic basket cells terminals. We conclude that the synaptic coexistence of vesicular glutamate and GABA transporters allows for corelease of both glutamate and GABA from selected nerve terminals, which may prevent systemic overexcitability by downregulating synaptic activity. Furthermore, our data suggest that VGLUT enhances transmitter storage in nonglutamatergic neurons. Thus, synaptic and vesicular coexistence of VGLUT and VGAT is more widespread than previously anticipated, putatively influencing fine-tuning and control of synaptic plasticity.

\section{Introduction}

Synaptic vesicles (SVs) concentrate neurotransmitters and release them by $\mathrm{Ca}^{2+}$-mediated membrane fusion upon presynaptic depolarization. Transmitter uptake by SVs requires an electrochemical gradient over the vesicular membrane provided by a vacuolar $\mathrm{H}^{+}$-ATPase and is mediated by specific vesicular transporters comprising VGLUT1, VGLUT2, and VGLUT3 for glutamate and VGAT/VIAAT for GABA (Edwards, 2007). In the CNS, the presence of either VGLUT1/2 or VGAT defines excitatory and inhibitory neurons, respectively. VGLUT3, however, mainly occurs in nonglutamatergic neurons, where it probably functions as a cotransmitter transporter (Gras et al., 2008; Seal et al., 2008). VGLUT1 and VGLUT2 are complementarily distributed and account for vesicular glutamate loading in the majority of glutamatergic neurons in the CNS. VGLUT1 is preferentially expressed in the neocortex and hippocampus, while VGLUT2 mainly occurs in the thalamus. VGLUT2 is the first transporter to be expressed before and after birth in rodents, while the definitive expression pattern for both VGLUT isoforms is achieved during postnatal development. This may explain the vesicular coexist-

Received Jan. 10, 2010; accepted Feb. 19, 2010.

Work in the authors' laboratories was supported by the Deutsche Forschungsgemeinschaft (G.A.H.) and Consejo Nacional de Ciencia y Tecnología (R.G.). We thank Marion Möbes and Annemarie Löchner for expert technical assistance and Allistair Garrat for linguistic corrections.

Correspondence should be addressed to Gudrun Ahnert-Hilger, AG Functional Cell Biology, Institute for Integrative Neuroanatomy, Charité Centrum 2 für Grundlagenmedizin, Philippstrasse 12, 10115 Berlin, Germany. E-mail: gudrun.ahnert@charite.de.

DOI:10.1523/JNEUROSCI.0141-10.2010

Copyright $\odot 2010$ the authors $\quad 0270-6474 / 10 / 307634-12 \$ 15.00 / 0$ ence of both VGLUT isoforms at least in some vesicle types (Herzog et al., 2006).

While the functioning of excitatory and inhibitory nerve terminals is based upon the strict segregation of VGLUT1/2 and VGAT expression (Edwards, 2007), there is mounting evidence that in subsets of neurons, VGLUT1/2 and VGAT may be present in the same nerve terminals. For instance, an increasing body of evidence suggests that both VGLUT1 and VGLUT2 colocalize in synapses of a select subset of neurons. VGLUT1 and VGAT (Kao et al., 2004; Gómez-Lira et al., 2005), and VGLUT2 and VGAT (Ottem et al., 2004), were found to colocalize by immunofluorescence microscopy. In addition, both VGLUT2 and VGAT were detected in a subset of presynaptic terminals in the rat hippocampus using postembedding immunogold electron microscopy (Boulland et al., 2009). Using a similar approach, we found both VGAT and VGLUT2 on small synaptic-like microvesicles within identical hormone-storing terminals of the rat neurohypophysis (I. Pahner, J. Klank, C. Frahm, J. Zander, M. Höltje, G. AhnertHilger, unpublished work).

The significance of coexpression of vesicular transporters for both excitatory and inhibitory transmitters in the same synapse is only beginning to emerge. For instance, electrophysiological experiments suggested that GABA is coreleased from glutamatergic hippocampal mossy fibers (Gutiérrez and Heinemann, 2001; Walker et al., 2001; Kasyanov et al., 2004) [see Uchigashima et al. (2007) for an alternative view]. Furthermore, it has been suggested that coexpression of a vesicular glutamate transporter (VGLUT3) in a cholinergic neuron may improve vesicular acetylcholine transporter (VAChT)-mediated vesicular acetylcho- 
line uptake and storage (Gras et al., 2008). However, it is unclear to what extent such functional cross talk governs transmitter uptake by synaptic vesicles expressing both excitatory and inhibitory neurotransmitter transporters.

In the present study, we have investigated to what extent VGAT colocalizes with either VGLUT1 or VGLUT2 at the vesicular and synaptic level and how such coexistence influences neurotransmitter uptake. Using monospecific antibodies, we immunoisolated different synaptic vesicle populations characterized by one of the respective transporters. We show that there is significant overlap between VGLUT2 and VGAT, with VGLUT activity enhancing GABA uptake but also allowing for uptake of glutamate in parallel. Furthermore, we show by postembedding immunoelectron microscopy that VGAT is present in hippocampal and cerebellar mossy fibers, i.e., classical glutamatergic synapses expressing VGLUT1 and VGLUT2. Conversely, VGLUT2 was detected in GABAergic cerebellar basket cell terminals, i.e., in a classical inhibitory terminal. Together, these findings show that coexistence of vesicular glutamate and GABA transporters is more widespread than previously appreciated, thus adding another layer of fine-tuning in the control of synaptic plasticity.

\section{Materials and Methods}

\section{Subcellular fractionation}

As starting material for immunoisolation, synaptosomes from adult rat whole brains were prepared as described previously (Becher et al., 1999). Briefly, brains were homogenized (Wheaton, Potter-Elvejhem, clearance 0.1-0.15 $\mu \mathrm{m}$ ) in ice-cold buffer [320 mm sucrose, $4 \mathrm{~mm} \mathrm{HEPES/KOH,}$ $\mathrm{pH}$ 7.4, $1 \mathrm{~mm}$ PMSF, and $1 \mu \mathrm{l} / \mathrm{ml}$ protease inhibitor cocktail (Pi, SigmaAldrich)] and centrifuged for $10 \mathrm{~min}$ at $4^{\circ} \mathrm{C}$ and $1300 \times g$ (Beckman rotor TLA-100.4). The resulting supernatant 1 (S1) was centrifuged again at $14,000 \times g$ for $15 \mathrm{~min}$, yielding a synaptosome-containing pellet (P2). The synaptosomes were osmotically shocked by diluting them 1:10 in lysis buffer (10 mM HEPES/KOH, pH 7.4, $1 \mathrm{~mm} \mathrm{PMSF,} \mathrm{and} 1 \mu \mathrm{l} / \mathrm{ml} \mathrm{Pi}$ ) and mechanically disrupted by three strokes at $2000 \mathrm{rpm}$ (PotterElvejhem, clearance $0.1-0.15 \mu \mathrm{m}$ ). These lysed synaptosomes (LS0) were used for immunoisolation. For neurotransmitter uptake experiments, immunoisolated subpopulations were taken from the lysis supernatant 1 (LS1) prepared from LS0 by a centrifugation step at 30,000 $\times g$ for $20 \mathrm{~min}$ at $4^{\circ} \mathrm{C}$. Some uptake experiments were performed with the usual synaptic vesicle fraction (LP2) prepared as given (Winter et al., 2005).

\section{Immunoisolation}

Superparamagnetic beads (Dynabeads Pan Mouse IgG, Invitrogen) were incubated for $2 \mathrm{~h}$ at $4^{\circ} \mathrm{C}$ in coating buffer [PBS, pH 7.4, 0.1\% BSA (w/v)] supplemented with $0.5-1 \mu \mathrm{g}$ of IgG per $10^{7}$ beads of one of the following primary mouse antibodies, all purchased from Synaptic Systems (catalog number given in parentheses), and directed against the following: synaptophysin (Syp, 101001 ), the vesicular glutamate transporter 1 (VGLUT1, 135 511) and 2 (VGLUT2, 135 411), the vesicular GABA transporter (VGAT, 131 011), or Rab 5 (108011).

The specificity of the transporter antibodies was checked by performing immunoisolations in either the presence or the absence of the respective peptides used for generation of the antibody. The peptides (VGLUT1: 135-3P, VGLUT2: 135-4P, VGAT: 131-0P) were obtained from Synaptic Systems. The coated beads were washed four times in coating buffer and then incubated overnight at $4^{\circ} \mathrm{C}$ with the LS0 fraction suspended in incubation buffer [PBS, pH 7.4, 2 mM EDTA, and 5\% BSA $(\mathrm{w} / \mathrm{v})]$ at a ratio of $75 \mu \mathrm{g}$ of protein to $1.4 \times 10^{7}$ beads. SVs bound to beads were washed three times in incubation buffer, followed by three washes in coating buffer, and finally dissolved in Laemmli buffer (Takamori et al., 2000). Beads without primary antibodies were subjected to the same procedure and used as control for nonspecifically bound material.

In some experiments (uptake of neurotransmitters into immunoisolated SVs), M-280 sheep anti-mouse beads (Invitrogen) were used instead, since they yielded lower background for the tritium-labeled neurotransmitters used in the uptake studies. Beads coupled with nonspecific mouse IgG (Santa Cruz Biotechnologies) served as control.

\section{SDS-PAGE/Western blot analysis}

The protein pattern of immunoisolated SVs was analyzed by an SDSPAGE/Western blot procedure using the following antibodies for subsequent immunodetection: guinea pig anti-glutamate transporter GLAST (AB 1782) and GLT1 (AB 1783) and rabbit anti-glutamate decarboxylase 65 (GAD65) (AB5082) and -GAD67 (MAB5992), purchased from Millipore Bioscience Research Reagents; mouse anti-AP2 (MA3-061), purchased from Dianova; mouse anti-synaptobrevin (104 211) and rabbit anti-VGLUT1 (135 302), -VGLUT2 (135 402) -VGLUT3 (135 203), -VGAT (131 002), -synaptogyrin (103 002), -synaptophysin (101 002), and -AP3 (155 003), all purchased from Synaptic Systems, and rabbit anti-rab5 (KAP-GP006), purchased from Stressgen Bioreagents. The specificity of the transporter antibodies was rechecked by preincubation with the peptides used for immunization (same control peptides for VGLUT1, VGLUT2, and VGAT as for immunoisolation). Immunosignals were visualized by either anti-mouse or anti-rabbit antibodies raised in horse or goat, respectively, and coupled to horseradish peroxidase (Vector Laboratories 94010). Stained bands were densitometrically analyzed using the LabImage 1D 2006 (Kapelan Bio-Imaging Solutions) and quantified using standard curves performed from the initial LS0 fraction.

\section{Neurotransmitter uptake}

Uptake assays were performed using SV-enriched lysis pellet (LP2) or immunoisolated SV subpopulations prepared from LS1 (see above). $\left[{ }^{3} \mathrm{H}\right]$ Serotonin uptake was performed as described previously (Brunk et al., 2006). Briefly, Syp-immunoisolated SVs or IgG-isolated SVs for control were suspended in potassium glutamate buffer [KG buffer containing $150 \mathrm{~mm}$ potassium glutamate; $20 \mathrm{~mm}$ 1,4-piperazinediethanesulfonic acid; 4 mM EGTA; 2.9 mM $\mathrm{MgCl}_{2}$ (is equivalent to $1 \mathrm{~mm}$ free $\mathrm{Mg}^{2+}$ ); and $2 \mathrm{~mm}$ ATP, adjusted to $\mathrm{pH} 7.0$ with $\mathrm{KOH}$ ]. Nonspecific serotonin uptake was estimated by reserpine and subtracted.

$\left[{ }^{3} \mathrm{H}\right]$ Glutamate uptake was performed using SVs or immunoisolated SV subpopulations prepared from LS1 (immunoisolated by anti-Syp, -VGLUT1, -VGLUT2, or -VGAT antibodies or IgG for control) with the exception that potassium gluconate buffer (KGC buffer, containing 150 mм potassium gluconate) was used instead as given earlier (Winter et al., 2005). Uptake was started by adding $49.5 \mu \mathrm{M} \mathrm{K}$-glutamate and $0.5 \mu \mathrm{M}$ $\left[{ }^{3} \mathrm{H}\right]$ glutamate to SVs. Nonspecific uptake was estimated using a combination of bafilomycin $(2 \mu \mathrm{M})$, nigericin $(5 \mu \mathrm{M})$, and valinomycin $(20 \mu \mathrm{M})$ and/or trypan blue $(1 \mu \mathrm{M})$, which specifically blocks VGLUT activity (Roseth et al., 1998).

Uptake of $\left[{ }^{3} \mathrm{H}\right] \mathrm{GABA}$ was performed in either KGC or KG buffer as indicated with $1 \mu \mathrm{M}\left[{ }^{3} \mathrm{H}\right] \mathrm{GABA}$ and $199 \mu \mathrm{M}$ unlabeled GABA. Nonspecific uptake was estimated using a combination of bafilomycin $(2 \mu \mathrm{M})$, nigericin $(5 \mu \mathrm{M})$, and valinomycin $(20 \mu \mathrm{M})$.

All radioactive neurotransmitters were purchased from GE Healthcare, and the unlabeled neurotransmitters were from Sigma-Aldrich.

Uptake was stopped after $10 \mathrm{~min}$ at $25^{\circ} \mathrm{C}$ by adding ice-cold $\mathrm{KG}$ or KGC buffer. Nonbound radioactivity was removed by centrifugation at $435,000 \times g($ Beckman rotor TLA-120.1) for $10 \mathrm{~min}$, and the pellets were washed three times. Radioactivity was measured by liquid scintillation counting (Beckman LS 6500, Beckman Coulter).

\section{Single-cell PCR}

The cytosolic content of electrophysiologically identified granule cells and interneurons was extracted as previously described (Gómez-Lira et al., 2005), applying gentle negative pressure with the pipette, and placed in a PCR tube containing the following: $8 \mu \mathrm{l}$ of buffer $5 \times$ (One-Step RT-PCR Kit, Qiagen), $1.6 \mu$ l of dNTPs (final concentration $10 \mathrm{~mm}$; OneStep RT-PCR Kit, Qiagen), 50 pmol of each oligonucleotide (to amplify the selected RNAs; see below), $40 \mathrm{U}$ of RNase inhibitor (Invitrogen), and $3 \mu \mathrm{l}$ of the mixture of reverse transcriptase "Omniscript," reverse transcriptase "Sensiscript," and DNA polymerase "HotStarTaq" (One-Step RT-PCR Kit, Qiagen), yielding a final volume of $40 \mu$ l. The RT-PCR was performed in a thermocycler (PCR express, Hybaid) as follows: $30 \mathrm{~min}$ at $50^{\circ} \mathrm{C} ; 15 \mathrm{~min}$ at $95^{\circ} \mathrm{C} ; 30 \mathrm{~s}$ at $94^{\circ} \mathrm{C}, 30 \mathrm{~s}$ at $52^{\circ} \mathrm{C}, 1 \mathrm{~min}$ at $72^{\circ} \mathrm{C}(40 \mathrm{PCR}$ cycles); and, finally, $10 \mathrm{~min}$ at $72^{\circ} \mathrm{C}$. A second PCR was then conducted 
with nested oligonucleotides. The product of the first amplifying proto$\operatorname{col}(3-5 \mu \mathrm{l})$ was used as template for a second PCR in a final volume of 25 $\mu$ l. The PCR solution contains the following: PCR buffer $10 \times(\mathrm{GE}$ Healthcare), $10 \mu \mathrm{M}$ dNTPs (Invitrogen), $1 \mathrm{U}$ of TaqDNA polymerase Taq (GE Healthcare), and $50 \mathrm{pmol}$ of each oligonucleotide. The reaction was performed as follows: $2 \mathrm{~min}$ at $94^{\circ} \mathrm{C}, 30 \mathrm{~s}$ at $94^{\circ} \mathrm{C}, 30 \mathrm{~s}$ at $55-58^{\circ} \mathrm{C}$, and 1 min at $72^{\circ} \mathrm{C}$ ( 35 PCR cycles). The specific oligonucleotides for each gene were custom designed (Invitrogen) based on the indicated references. They were as follows: for VGAT (McIntire et al., 1997), sense, 5'ATTCAGGGCATGTTCGTGCT3'; antisense, 5'ATGTGTGTCCAGTTCATCAT3'; nested, 5'TGATCTGGGCCACATTGACC3'; for VGLUT-1, sense, 5'ACGTGAACCACTTGGACATC3'; antisense, 5'CAAGAGGCAGTTGAGAAGGA3'; nested, 5'CAGTACGTGTTCCTCATAGC3'. The products were visualized by electrophoresis in agarose gels (1.5\%) with ethidium bromide. The expected size of each final PCR product is VGAT 250 bp and VGLUT-1 450 bp, respectively.

\section{Electron microscopy}

Tissue preparation. For all procedures, ethical approval was obtained in accordance with the National Act on the Use of Experimental Animals (Germany). Male adult Wistar rats (300-350 g) from various animal suppliers (Haarlan Winkelmann and local animal breeding of the Charité) were anesthetized by a mixture of medetomidine $(500 \mu \mathrm{g} / \mathrm{kg}$, Pfizer, Orion Pharma) and ketamine ( $75 \mathrm{mg} / \mathrm{kg}$, Parke-Davis Pharmacia) and perfused through the left cardiac ventricle using a freshly prepared solution of $4 \%$ paraformaldehyde (Electron Microscopy Sciences), supplemented with $0.05 \%$ glutaraldehyde (Fluka Chemie) and $0.2 \%$ picric acid (Fluka Chemie) in $0.1 \mathrm{~m}$ sodium phosphate buffer (PB) at $\mathrm{pH}$ 7.4. The brains were removed, dissected, and postfixed overnight at $4^{\circ} \mathrm{C}$. Embedding and immunogold labeling followed a modification of a protocol given earlier (Pahner et al., 2003).

Freeze substitution embedding. Brain slices (1-2 mm thick) were prepared from hippocampus and cerebellum (vermis) and washed $6 \times 10$ $\min$ in $0.1 \mathrm{M} \mathrm{PB}$ at $\mathrm{pH}$ 7.4. The slices were cryoprotected stepwise in $\mathrm{PB}$ supplemented with increasing concentrations of glycerol $[10-20-30 \%$ $(\mathrm{v} / \mathrm{v})]$ and left overnight in $30 \%$ glycerol in $\mathrm{PB}$ at $4^{\circ} \mathrm{C}$. The tissue was frozen by plunging into hexane (Carl Roth) at a temperature of $-70^{\circ} \mathrm{C}$. The samples were transferred into cold methanol $\left(-90^{\circ} \mathrm{C}\right)$ in a freezesubstitution chamber (Leica EM AFS). The methanol was exchanged three times before the specimens were immersed overnight in anhydrous methanol at $-90^{\circ} \mathrm{C}$, containing $2 \%(\mathrm{w} / \mathrm{v})$ uranyl acetate. After rinsing several times with methanol, the temperature was gradually raised to $-50^{\circ} \mathrm{C}$ and left overnight at $-50^{\circ} \mathrm{C}$. The tissue was then infiltrated with mixtures of Lowicryl HM20 resin (Polysciences) and methanol (1:2; 1:1; $2: 1,1 \mathrm{~h}$ each) and finally left in pure resin overnight at $-50^{\circ} \mathrm{C}$. The samples were transferred to flat embedding molds containing freshly prepared resin at $-50^{\circ} \mathrm{C}$. UV polymerization was started at $-50^{\circ} \mathrm{C}$ (overnight) and then continued for $4 \mathrm{~d}$ at temperatures gradually increasing from $-50^{\circ} \mathrm{C}$ to $-20^{\circ} \mathrm{C}(24 \mathrm{~h})$ and finally to $+20^{\circ} \mathrm{C}(24 \mathrm{~h})$. Ultrathin sections (70 $\mathrm{nm}$ ) were mounted on 200-mesh formvar-coated nickel grids (Plano).

Postembedding immunogold labeling. Primary antibodies against vesicular amino acid transporters were purchased from Synaptic Systems (catalog number and dilutions given in parentheses): rabbit anti-VGLUT1 (135302, 1:100), guinea pig anti-VGLUT1 (135304, 1:100), rabbit antiVGLUT2 (135402, 1:100), guinea pig anti-VGAT (131004, 1:50), rabbit anti-VGAT (131002, 1:100), and rabbit anti-VGAT first luminal domain (131103, 1:50). Two additional VGAT antibodies directed against the C-terminal part (amino acids 510-525) were generous gifts from $\mathrm{H}$. Martens (Synaptic Systems, Göttingen, Germany) (Martens et al., 2008) and diluted 1:100. A further guinea pig anti-VGAT directed against the C-terminal peptide amino acids 511-525 was purchased from Calbiochem (676780, 1:100). Rabbit anti-GAD65 (AB 5082, 1:100) and rabbit anti-GAD67 (AB 5992, 1:100) were obtained from Millipore Bioscience Research Reagents.

All postembedding steps except for the incubation with primary antibodies were performed at room temperature. For single and double immunolabeling, sections ( $70 \mathrm{~nm}$ thick) were first incubated two times for 5 min in 0.1 M PBT (PBS, $0.001 \%$ Triton X-100, 0.001\% Tween 20, pH 7.4), followed by 90 min incubation in PBT supplemented with $2 \%$ BSA (bovine serum albumin, Sigma-Aldrich) and 5\% NGS (normal goat serum, PAN Biotech) at room temperature. The sections were next incubated with primary antibodies diluted in the same buffer overnight at $4^{\circ} \mathrm{C}$ in a humid chamber. After rinsing several times with PBT, the binding of primary antibodies was visualized by incubating with goat anti-rabbit or goat anti-guinea pig secondary antibodies conjugated to either 5 or 10 $\mathrm{nm}$ gold particles (British BioCell, International) in PBT supplemented with $0.5 \%$ acetylated BSA (Aurion), for $70 \mathrm{~min}$ in a humid chamber. Grids were rinsed several times in PBT, PBS, and finally in water. Thin sections were stained with $2 \%$ aqueous uranyl acetate (Merck) for $2 \mathrm{~min}$, and with lead citrate for $30 \mathrm{~s}$. Sections were examined using a Zeiss EM 900 equipped with a digital camera (Proscan $1 \mathrm{~K}$ Slow-Scan CCD-Camera).

For negative controls, primary antibodies were omitted. Specificity of VGAT immunoreactivity was additionally verified by preincubation with the respective antigen used for immunization (see supplemental material, available at www.jneurosci.org).

Quantification of immunogold signals. Hippocampal or cerebellar sections were incubated with the respective transporter antibodies, and immunogold labeling was detected by secondary antibodies coupled to either $10 \mathrm{~nm}$ (hippocampus) or $15 \mathrm{~nm}$ (cerebellum) gold particles. Quantification was performed using the Esivision Pro 3.2 software (Soft Imaging System).

\section{Results}

\section{Transporter-specific immunoisolation of} vesicle subpopulations

Proteomic analysis of SVs obtained by transporter-specific immunoisolation revealed that VGLUT- and VGAT-containing vesicles are mostly similar with respect to protein profile. Thus, these transporters appear to be sufficient to define the neurotransmitter phenotype of SVs in general. Apart from defining either excitatory or inhibitory axon terminals, VGLUT and VGAT may also occur at the same SVs in subtypes of GABAergic and glutamatergic terminals, modulating vesicular content as suggested for VGLUT3 (Gras et al., 2008; Seal et al., 2008). To see whether a vesicular colocalization of VGAT and VGLUT exists in a significant fraction of a whole-brain SV preparation, we performed immunoisolations using monoclonal antibodies directed against VGLUT1, VGLUT2, and VGAT. In addition, we also immunoisolated SVs with antibodies against Syp as control for the entire SV population.

As expected, all VGLUT isoforms as well as VGAT could be detected on Syp immunoisolates from different preparations. When analyzing the VGLUT1- and VGLUT2-specific immunoisolates, the previously observed coexistence of both transporters (Herzog et al., 2006) could be confirmed for adult animals. Surprisingly, VGAT was seen in VGLUT2 immunoisolates as well as VGLUT2 in VGAT immunoisolates (Fig. 1A).

The relative amounts of neurotransmitter transporters and the abundant vesicular proteins Syp, synaptogyrin (Syg), and synaptobrevin (Syb) were quantified using vesicular subpopulations immunoisolated with antibodies against VGLUT1, VGLUT2, and VGAT. Immunoisolations performed either with the Syp antibody or in the absence of any antibody served as positive and negative controls, respectively. A standard curve for the proteins to be detected was performed using the LSO fraction, the starting material for the immunoisolation (Fig. $1 A$, left). For each transporter immunoisolate, relative amounts of the given proteins were calculated from three different immunoisolations. The amount of VGAT on VGLUT2 immunoisolates was significantly higher than the fraction isolated by VGLUT1 antibodies (Fig. 1A, diagram). Also, VGLUT1 immunoisolates apparently associated with higher amounts of the tetraspan proteins Syp and Syg than VGLUT2 or VGAT immunoisolated subpopulations (Fig. 1A) (ratio of Syp plus Syg to VGLUT1: $1.20 \pm 0.29$; ratio of 
A LS0 starting material $[\mu \mathrm{g}]$
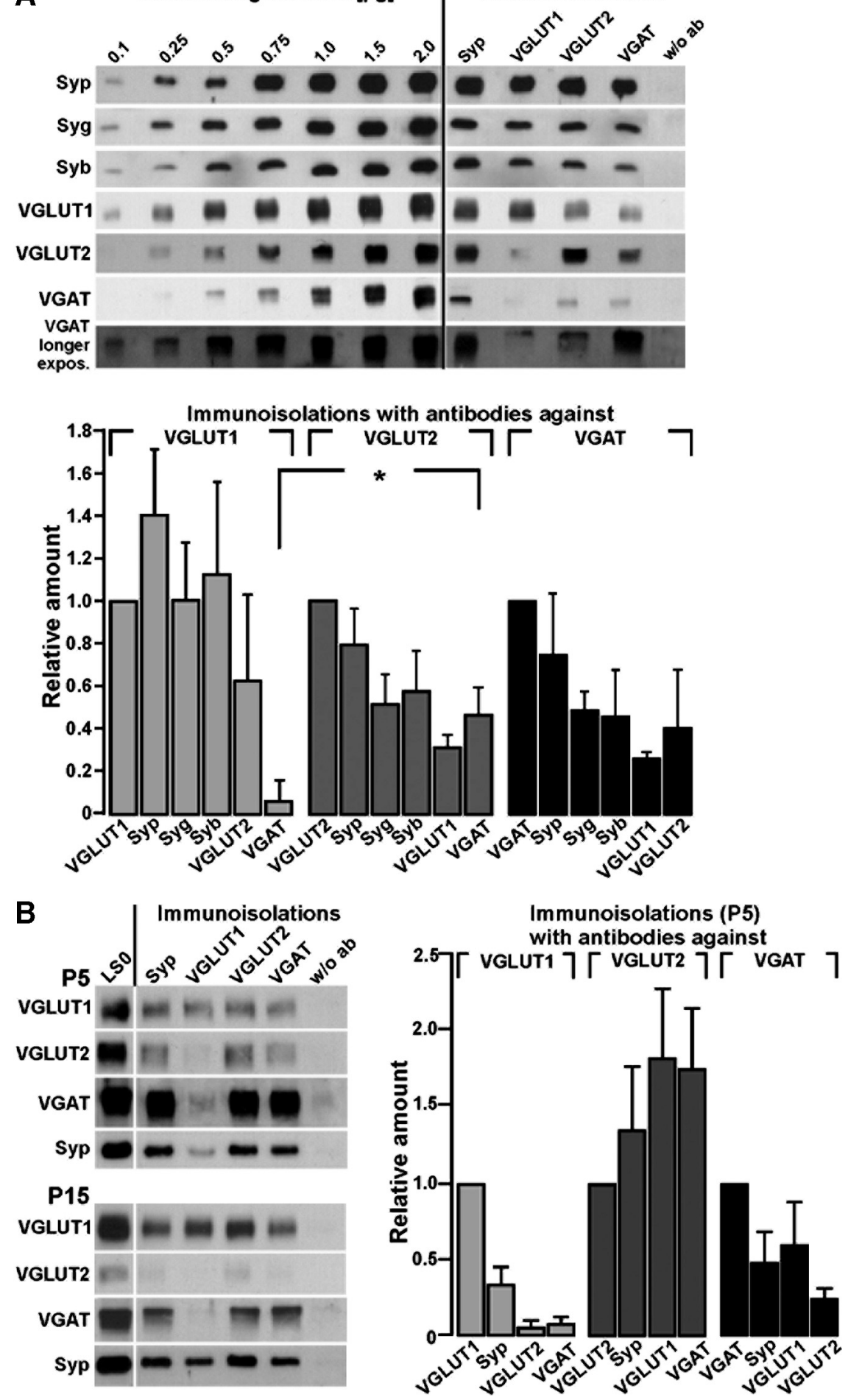

Figure 1. Comparison of the amounts of abundant vesicular proteins and transmitter transporters on SVs immunoisolated from adult or juvenile rat brains by antibodies against VGLUT1, VGLUT2, and VGAT. A, Immunoisolated vesicles (adult brains) were obtained as given in Materials and Methods and detection was performed with the antibodies indicated or without antibody (w/0 ab). Detection of the vesicular proteins indicated on the left side was performed using the respective polyclonal rabbit antibodies. A standard curve from the starting material ( $(\mathrm{SO})$ ) was performed in parallel to calculate the respective protein amounts in the individual immunoisolates. Relative amounts of Syp, Syg, and Syb as well as of VGLUT1, VGLUT2, and VGAT were quantified from three different immunoisolation experiments using standard curves performed from the initial LSO fraction. Note that VGLUT2immunoisolated vesicles harbor more VGAT than VGLUT1 isolates ( ${ }^{*}$ Student's $t$ test, $p<0.03$ ). $\boldsymbol{B}$, Transporter-specific immunoisolated vesicles were obtained as given in methods using lysed synaptosomes (LSO) from rat brains at postnatal days 5 and 15 . The amount of VGAT on VGLUT2-immunoisolated vesicles was the highest at P5. Relative amounts of Syp, VGLUT1, VGLUT2, and VGAT were quantified from three different immunoisolation experiments using P5 brains. Standard curves from the initial LSO fraction of P5 brains were performed as in $\boldsymbol{B}$ (data not shown); the LSO fraction presented in the graph corresponds to $4 \mu \mathrm{g}$ of protein. Blots were developed for $30 \mathrm{~s}$ or $3 \mathrm{~min}$ (longer exposure).
Syp plus Syg to VGLUT2: $0.66 \pm 0.17$; ratio of Syp plus Syg to VGAT: $0.62 \pm$ $0.20 ; p=0.009$ or 0.007 , respectively). The slight variations in the amounts of Syb were not significant.

Faint signals for Syp and the neurotransmitter transporters were seen on isolates using an antibody against the endosomal marker Rab5 used as control for endosomal membranes. Accordingly, Rab5 yielded only very faint signals in all immunoisolates (supplemental Fig. 1A, available at www.jneurosci.org as supplemental material). In addition, we detected VGLUT3 in immunoisolates of Syp, VGLUT2, and VGAT (supplemental Fig. $1 A$, available at www.jneurosci.org as supplemental material). Immunoisolates may also contain endocytic vesicles derived from membrane patches of different origin and thus falsely indicate a colocalization on the same SVs. Therefore we also tested for the adaptor proteins (AP2 and AP3) involved in different endocytic pathways, respectively. While low amounts of AP2 were found in all immunoisolates, there was no detectable signal for AP3 (supplemental Fig. $1 B$, available at www. jneurosci.org as supplemental material). To check for plasma membrane proteins, we analyzed the plasma membrane transporter GLT1 and GLAST. GLT1 has also been detected in neurons (Chen et al., 2004) besides its occurrence in astrocytes. We found small amounts of GLT1 especially in VGLUT2 immunoisolates. In contrast, GLAST, another glutamate transporter preferentially occurring in astrocytes was not detected in any of the immunoisolates underscoring the specificity and sensitivity of the immunoisolation procedure. As expected, the vesicle-associated GABA-synthesizing enzyme GAD67 was observed in VGAT immunoisolates, while the GAD65 isoform was not detected on vesicular immunoisolates (supplemental Fig. $1 B$, available at www. jneurosci.org as supplemental material). Further confidence in the specificity of the antibodies against the various transporters used for Western blot detection (all raised in rabbits) and for immunoisolation (all raised as monoclonals) was obtained by preincubating with the respective peptides (supplemental Fig. $1 C, D$, available at www.jneurosci.org as supplemental material).

We next analyzed the developmental expression of the vesicular glutamate and GABA transporters. As expected, during postnatal development VGLUT1 expression increased more than the expression of VGLUT2 and VGAT. This was also ev- 
A ${ }^{1800}$ Serotonin Glutamate
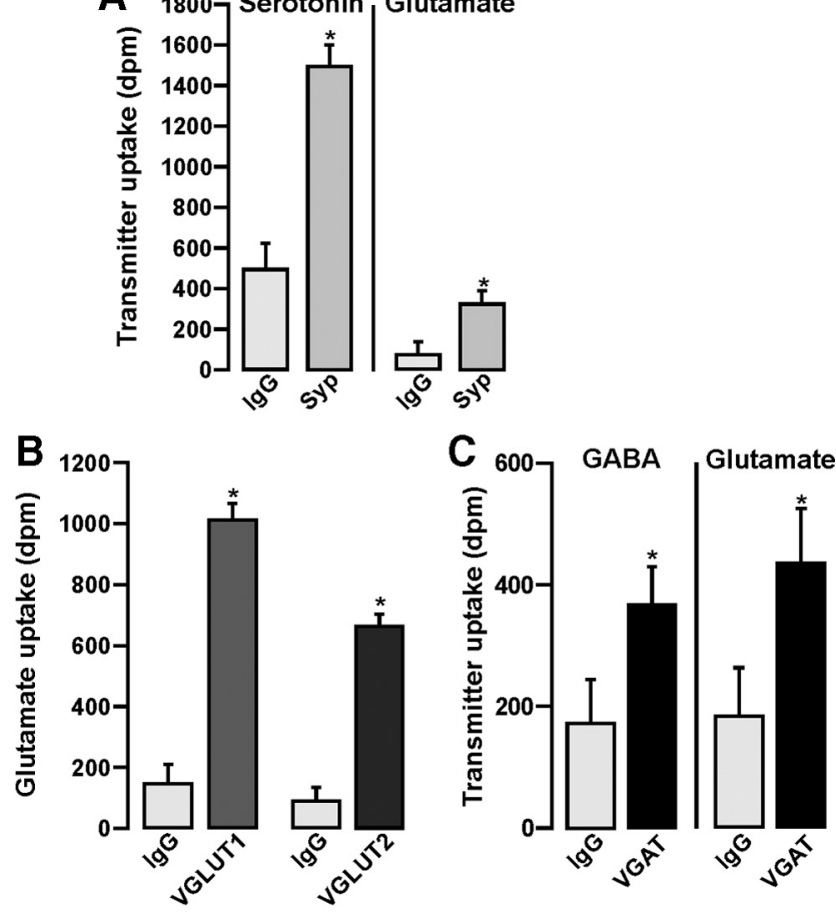

D

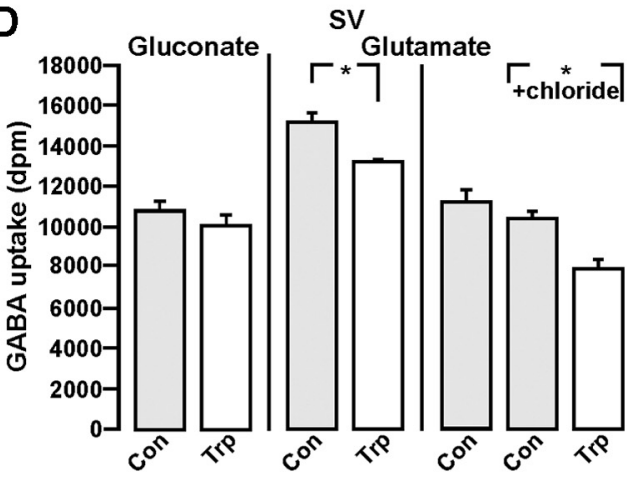

E

SV

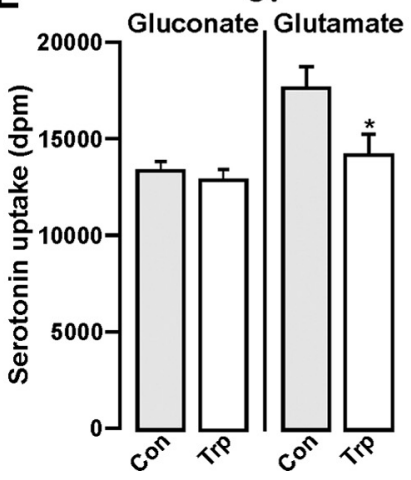

Figure 2. Transmitter uptake into SVs and SV subpopulations. $A,\left[{ }^{3} \mathrm{H}\right]$ Serotonin or $\left[{ }^{3} \mathrm{H}\right] \mathrm{glu}-$ tamate uptake was performed using SVs immunoisolated without (lgG) or with (Syp) the Syp antibody. $\boldsymbol{B},\left[{ }^{3} \mathrm{H}\right] \mathrm{Glutamate}$ uptake was performed using SVs immunoisolated without (IgG) or with an antibody against either VGLUT1 or VGLUT2. $C,\left[{ }^{3} H\right]$ GABA or $\left[{ }^{3} \mathrm{H}\right]$ glutamate uptake was performed using SVs immunoisolated without (IgG) or with (VGAT) the VGAT antibody. Note that VGAT immunoisolates take up GABA and glutamate. $\boldsymbol{D},\left[{ }^{3} \mathrm{H}\right] \mathrm{GABA}$ uptake was performed into SVs (LP2 fraction) suspended in either gluconate containing KGC buffer or glutamate containing KG buffer in the absence (Con) or presence of $5 \mu \mathrm{m}$ trypan blue (Trp). To avoid dilution of $\left[{ }^{3} \mathrm{H}\right] \mathrm{GABA}$ by endogenously generated GABA, an inhibitor of GAD, 3-mercapto-propionic acid $(10 \mu \mathrm{M})$, was present throughout. There was no Trp-mediated inhibition of uptake in KGC buffer (Gluconate, left columns). GABA uptake was significantly decreased (13\%) in KG buffer (Glutamate) and further decreased (24\%) when glutamate was partially replaced by $50 \mathrm{~mm}$ chloride ident in the respective immunoisolations. At postnatal day 5 (P5), considerable amounts of VGAT were seen in subpopulations immunoisolated by VGLUT and, in turn, VGAT immunoisolates contained VGLUT2 and also VGLUT1 as revealed by quantification (Fig. $1 B$ ). At P15, there was still a considerable degree of colocalization between VGLUT2 and VGAT, which correlates well with the higher amount of VGAT mRNA found in isolated hippocampal granular cells at P15 (see below). At P30, the ratios were already similar to those found in adult animals (data not shown).

\section{Contribution of VGLUT transport activity to monoamine and} GABA uptake

VGLUT2 was found on GABAergic vesicles (see above), and VGLUT3 has been observed in "nonglutamatergic" serotoninstoring terminals (Gras et al., 2008). Furthermore, VGLUT3 activity improves VAChT-mediated acetylcholine uptake and storage (Gras et al., 2008). We therefore asked whether VGLUT activity (VGLUT3 and/or VGLUT2) can improve the vesicular loading of GABA by VGAT and also, as another control, of serotonin by VMAT2. To this end, we first analyzed transmitter uptake of immunoisolated vesicles using $\left[{ }^{3} \mathrm{H}\right]$-labeled glutamate, $\mathrm{GABA}$, and serotonin. Serotonin is transported by the vesicular monoamine transporter VMAT2. Hence, VMAT2-containing SVs should be present in immunoisolates using the Syp antibody. Syp immunoisolates specifically took up serotonin or glutamate, while the respective control beads, loaded with nonspecific mouse Ig (IgG), accumulated less radioactivity (Fig. 2A). Glutamate uptake was increased in VGLUT1 or VGLUT2 immunoisolates compared to Syp isolates (Fig. $2 A, B$ ). We also observed a significant GABA uptake when using VGAT-immunoisolated vesicles (Fig. 2C). VGAT immunoisolates took up glutamate in addition to GABA, thus supporting a vesicular colocalization of VGAT and VGLUT (Fig. 2C). GABA uptake, which is generally difficult to measure due to the very low affinity of VGAT for its substrate, was not demonstrable with VGLUT immunoisolates, presumably reflecting a relatively small subset of vesicles in this preparation containing both VGLUT and VGAT. To overcome this limitation, we analyzed the putative contribution of VGLUT activity to VGAT-mediated GABA uptake by using a SV preparation from whole brain and the specific VGLUT inhibitor trypan blue (Roseth et al., 1998). As previously shown, trypan blue selectively inhibits glutamate uptake comparable to an inhibition by dissipating the electrochemical gradient (Winter et al., 2005). In the presence of glutamate, trypan blue inhibited GABA uptake by $15 \%$, indicating that VGLUT activity can improve GABA uptake. No contribution of VGLUT activity was seen in the complete absence of glutamate, when gluconate as major anion replaced glutamate, indicated by the lack of an inhibition by trypan blue (Fig. $2 D$ ). These data indicate that a subpopulation of GABAergic vesicles contains one of the VGLUT isoforms. The inhibition by trypan blue was even more pronounced when the chloride concentration was increased to $50 \mathrm{~mm}$ while glutamate concentration was decreased, respectively, which only marginally affected GABA uptake in general (Fig. 2D). This effect may be

\section{$\longleftarrow$}

(Glutamate + chloride), indicating a contribution of VGLUT activity. $\boldsymbol{E},\left[{ }^{3} \mathrm{H}\right]$ Serotonin uptake into SVs was performed in the absence (Con) or presence of Trp, either in KGC buffer (gluconate) or in KG buffer (glutamate). Trp significantly diminished serotonin uptake in KG buffer, indicating a contribution of VGLUT activity. Uptakes are given in dpm (decay/min) and were corrected for nonspecific uptake in the presence of $2 \mu \mathrm{m}$ reserpine (serotonin uptake) or $2 \mu \mathrm{m}$ bafilomycin (GABA or glutamate uptake). Values represent the mean of three determinations \pm SD ( ${ }^{*}$ Student's $t$ test, $\left.p<0.05\right)$. 
due to the increased chloride gradient over the vesicular membrane, which favors VGLUT activity (Schenck et al., 2009). Similarly, VMAT2-mediated serotonin transport was also improved by VGLUT activity. Here a $15 \%$ reduction of serotonin uptake was observed in the presence of trypan blue. Again, this effect required the presence of glutamate as the major anion (Fig. 2E).

\section{Synaptic coexistence of VGAT with VGLUT1 and/or VGLUT2} Given that a subpopulation of SVs apparently contains more than one type of transmitter transporter and that VGLUT activity contributes to GABA uptake, we next set out to analyze the synaptic coexistence of VGLUT1/VGLUT2 and VGAT in more detail at the mRNA and electron microscopic level.

\section{VGAT mRNA expression in granule cells of the hippocampal} dentate gyrus

The presence of GABA-synthesizing enzymes GAD65/67 and GABA has been documented in bona fide glutamatergic hippocampal granule cells of the dentate gyrus. The observed GABAergic "phenotype" is increased in these cells during development and following repeated stimulation (Sloviter et al., 1996) (for review, see Gutiérrez, 2005). However, although the presence of VGAT mRNA in granule cells and in mossy fiber synaptosomes has been described (Gómez-Lira et al., 2005), direct evidence of VGAT protein expression in glutamatergic cells i.e., in dentate gyrus granule cells is still lacking. Therefore acutely isolated hippocampal granule cells of the dentate gyrus from either postnatal day $15(n=15)$ or adult $(n=15)$ rats were identified by electrophysiological recordings and the collected cytoplasm was subjected to single-cell PCR using primers specific for VGLUT1 and VGAT. The same procedure was applied to interneurons $(n=3)$ of the dentate gyrus for comparison. Clear and almost equally strong bands for both transporters were observed at P15 (Fig. 3A), also correlating with the vesicular colocalization of VGAT and VGLUT at earlier postnatal states (Fig. 1). Afterward the VGAT expression declined, while as expected, VGLUT1 was highly expressed at P30. However, a faint signal for VGAT mRNA was still present in $\sim 40 \%$ of the adult granule cells analyzed (Fig. $3 B)$. These data reflect the results obtained by immunoisolation (see above) and also correlate with the postembedding electron microscopic analysis (see below).

Electron microscopic evidence for VGAT in glutamatergic mossy fiber terminals of the hippocampus

Immunoisolation experiments do not discriminate between glutamate-containing but morphologically different synaptic terminals like CA1 Schaffer collateral terminals and CA3 mossy fibers, which should not necessarily be equipped with the same set of additional neurotransmitter transporters. We therefore postulated that the coexistence of VGLUT and VGAT within the same axon terminals may be more likely in large terminals, establishing multiple synaptic contacts, such as hippocampal mossy fiber terminals. This prompted us to perform postembedding immunogold labeling on hippocampal tissue, focusing on glutamatergic mossy fiber terminals.

As expected, mossy fiber terminals in the CA3 area from adult rat hippocampus were robustly labeled for VGLUT1 (Fig. 4A, C) and moderately labeled for VGLUT2 (Fig. 4B). However, the same type of terminal was also clearly immunopositive for VGAT (Fig. 4D). In comparison to VGLUT1, the VGAT immunoreactivity was generally less pronounced, even in GABAergic terminals establishing symmetric contacts on pyramidal cell bodies (supplemental Fig. 2 B, available at www.jneurosci.org as supplemental material). Thus, the moderate strength of VGAT immu-
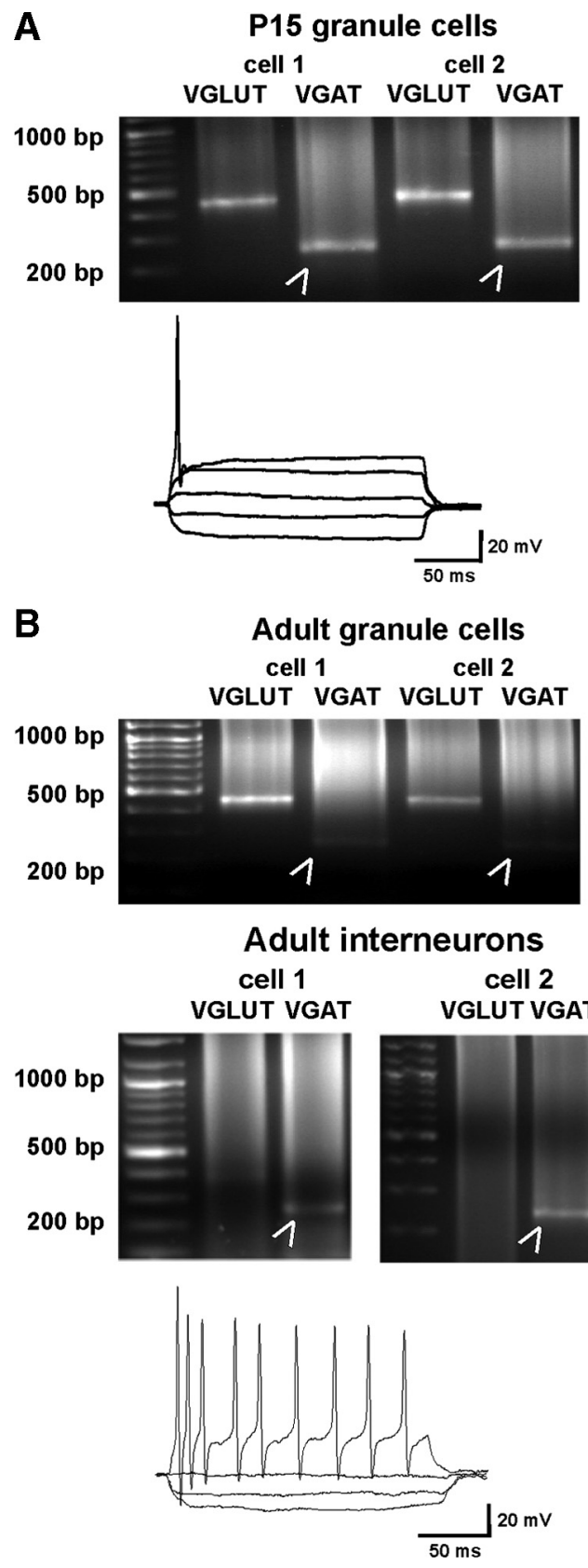

Figure 3. Identification of VGAT mRNA in hippocampal granular cells. $A$, Hippocampal granule cells of P15 and adult rats were identified electrophysiologically, based on their typical single action potential following depolarizing pulses, and single-cell $P C R$ was performed using primers specific for VGLUT1 or VGAT. All granule cells at P15 (2 examples are shown) expressed both VGLUT1 and VGAT (arrowheads). Approximately $40 \%$ of the adult granule cells ( 15 cells in total; 2 examples are shown) showed a low expression of VGAT in addition to VGLUT1 (arrowheads). $\boldsymbol{B}$, For comparison, interneurons isolated from the dentate gyrus and the hilar region were identified electrophysiologically. Interneurons typically fire repetitive action potentials throughout the depolarizing pulse without presenting accommodation. Single PCR was performed using both primers. In the two examples shown, a clear band for VGAT (arrowheads) was present, while VGLUT1 was completely absent.

noreactivity in glutamatergic terminals possibly underestimates to some degree the relative amount of VGAT occurring in these terminals. To further support the specificity of the VGAT immunoreaction, and because several groups have failed to find VGAT immunoreactivity in the hippocampal mossy fiber terminals (Chaudhry et al., 1998; Sperk et al., 2003), we tested a variety of VGAT antibodies raised in different species and directed against 
the $\mathrm{C}$ terminus, the first luminal domain, or the $\mathrm{N}$ terminus of the transporter. In the CA3 region, all antibodies resulted in a comparable labeling of GABAergic terminals characterized by their symmetric contacts. In addition, large terminals classified as glutamatergic mossy fiber terminals due to their size, asymmetric contacts, and occurrence in the stratum lucidum were also immunopositive (see supplemental Figs. $2-4$, available at www.jneurosci.org as supplemental material). Preincubation with the fusion protein or the peptide used for immunization completely abolished the specific immunosignals obtained with VGAT antibodies (see supplemental Figs. 2, 3, available at www.jneurosci.org as supplemental material). In contrast to mossy fiber terminals, small glutamatergic terminals synapsing on dendritic spines in stratum radiatum [supplemental Fig. $2 B$ (indicated by thin arrows), available at www.jneurosci.org as supplemental material] did not show any labeling at all. As expected from the singlelabeling experiments as shown in Figure $4 D$, VGAT colocalized with VGLUT1 (Fig. $4 E$, detail in $G$ ) and VGLUT2 (Fig. $4 F$, detail in $H$ ) in hippocampal mossy fiber terminals, indicating a mixed glutamatergic/GABAergic phenotype of these terminals. However, we did not observe any obvious segregation of VGLUT or VGAT to different areas within the terminal. This correlates with the scattered distribution of GABA and glutamate in the same terminals (Bergersen et al., 2003).

Occasionally, we observed VGAT- and VGLUT-specific immunogold particles associated with the same vesicle (Fig. $4 H$ ). However, since the length of the antibody bridge between epitope and gold particle becomes significant, limiting the resolution of the immunogold method to $\sim 20 \mathrm{~nm}$, a colocalization on the same vesicle is difficult to demonstrate unequivocally in areas containing densely packed synaptic vesicles such as in hippocampal mossy fibers. Nevertheless, our biochemical and functional findings (see above) strongly argue for a colocalization of VGLUT and VGAT on the same vesicle.

The GABAergic phenotype of hippocampal mossy fiber terminals was further supported by double immunolabeling for VGLUT1/2 and GAD65/67, the GABAsynthesizing enzymes. Both, GAD65 and GAD67 as cytosolic enzymes colocalized with VGAT in hippocampal mossy fiber terminals (supplemental Fig. 2C,D, available at www.jneurosci.org as supplemental material).

It is noteworthy that the labeling intensity for VGAT in CA3 mossy fibers varied considerably, with $\sim 30-70 \%$ of terminals being significantly immunopositive for VGAT, depending on the VGAT antibody used (see supplemental Figs. 2-4, Table 1, available at www.jneurosci.org as supplemental material).
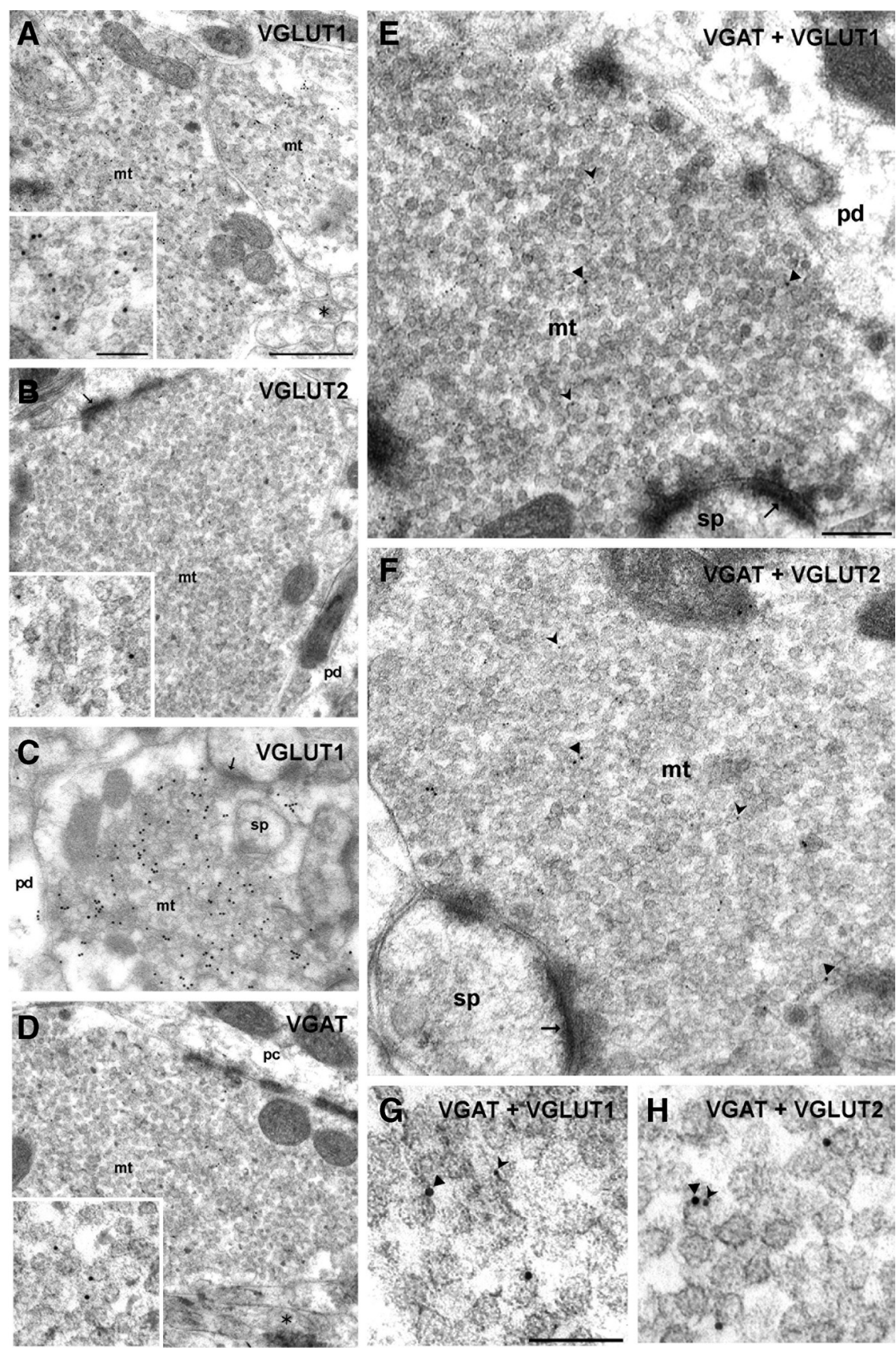

Figure 4. Single and double immunogold labeling for VGLUT1, VGLUT2, and VGAT in terminals of the hippocampal CA3 region. $A-C$, Mossy fiber terminals $(\mathrm{mt})$ are clearly labeled by rabbit antibodies directed againsteither VGLUT1 or VGLUT2 indicated by $10(A, B)$ or $15(C)$ $\mathrm{nm}$ gold particles. The asterisk indicates an axon bundle profile. Pd, Pyramidal cell dendrite. The insets in $\boldsymbol{A}$ and $\boldsymbol{B}$ represent details at a higher magnification. D, A mossy fiber terminal ( $\mathrm{mt}$ ) of the CA3 area of the hippocampus shows vesicle-associated immunoreactivity for the VGAT guinea pig antiserum indicated by $10 \mathrm{~nm}$ gold particles. The asterisk indicates an axon, $\mathrm{pc}$ pyramidal cell body. The inset represents a detail at a higher magnification. $\boldsymbol{E}-\boldsymbol{H}$, Mossy fiber terminals ( $\mathrm{mt}$ ) were double immunolabeled with the guinea pig antiserum againstVGAT ( $10 \mathrm{~nm}$ gold particles) and rabbit antisera against either VGLUT1 or VGLUT2 (both $5 \mathrm{~nm}$ gold particles). $\boldsymbol{G}$ and $\boldsymbol{H}$ represent micrographs at a higher magnification, with arrowheads indicating VGAT and forked arrowheads indicating either VGLUT1 (G) or VGLUT2 (H). Note the SVs exhibiting putatively colocalized immunogold signals for VGAT and VGLUT2 shown in $\boldsymbol{H}$. Thin arrows indicate asymmetric contacts. Sp, Spine; pd, pyramidal cell dendrite. Scale bars: $\boldsymbol{A}-\boldsymbol{D}, 500 \mathrm{~nm} ; \boldsymbol{E}, \boldsymbol{F}, 200 \mathrm{~nm} ; \boldsymbol{G}, \boldsymbol{H}$, insets in $\boldsymbol{A}, \boldsymbol{B}$, and $\boldsymbol{D}, 100 \mathrm{~nm}$.

\section{Coexistence of VGLUT and VGAT in different types of} cerebellar terminals

Since whole-brain preparations were used in our biochemical experiments, it was tempting to speculate that in addition to hippocampal mossy fiber terminals, other glutamatergic terminals, e.g., in the cerebellum, may also be equipped with VGAT. Similarly, VGLUT may be coexpressed in other types of GABAergic terminals. 

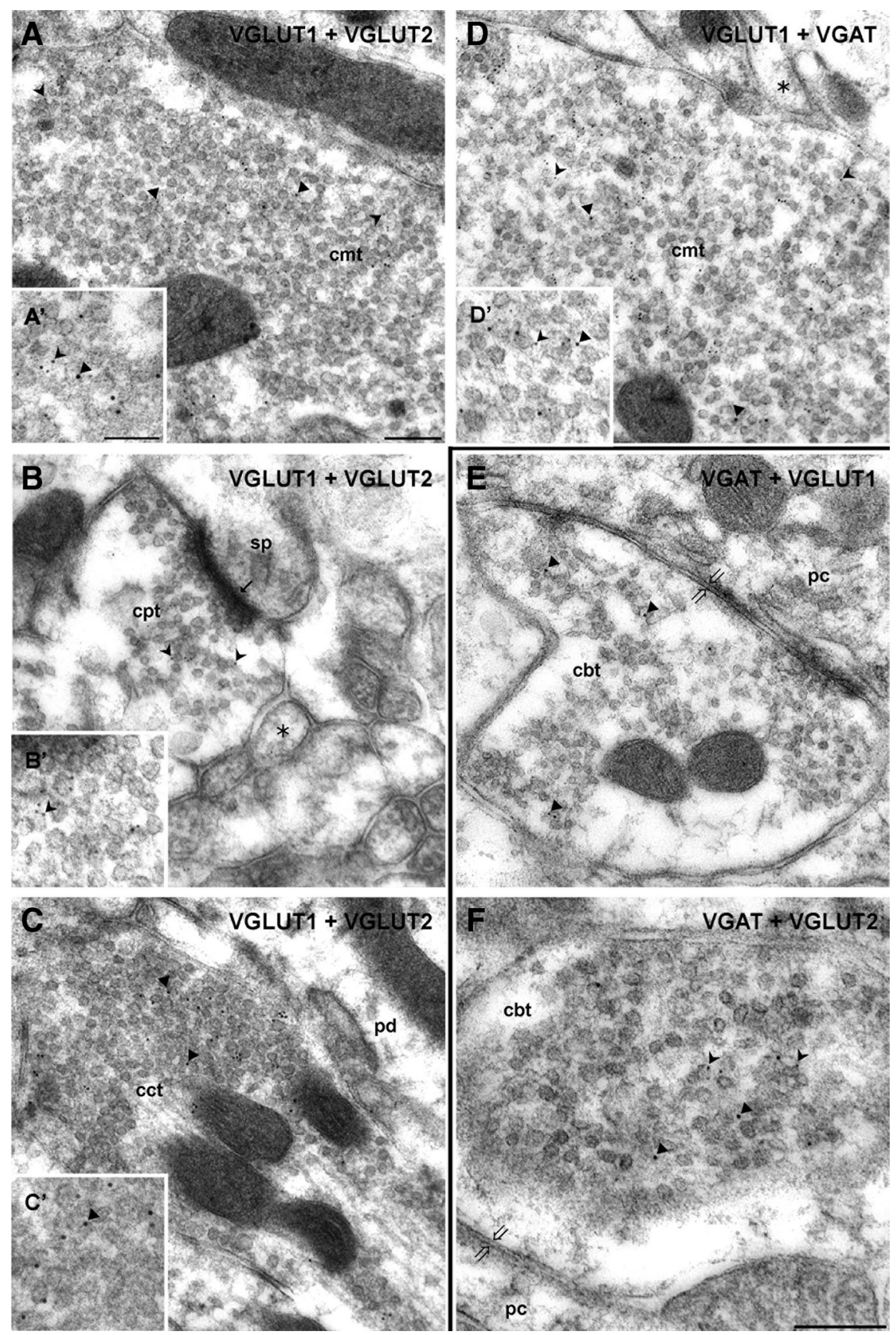

Figure 5. Double immunogold labeling for VGLUT1, VGLUT2, and VGAT in glutamatergic and GABAergic terminals of the cerebellar cortex. $\boldsymbol{A}$-C, Double immunogold labeling of VGLUT1 (guinea pig antiserum, $5 \mathrm{~nm}$ gold particles, forked arrowheads in $\boldsymbol{A}^{\prime}$ and $\boldsymbol{B}^{\prime}$ ) and VGLUT2 (rabbit antiserum, $10 \mathrm{~nm}$ gold particles, arrowheads in $A^{\prime}, C^{\prime}$ ) in a mossy fiber terminal of the granular cell layer $\left(\mathrm{cmt}, A, A^{\prime}\right)$, a parallel fiber terminal of the molecular layer ( $\left(\mathbf{c p t}, \boldsymbol{B}, \boldsymbol{B}^{\prime}\right)$, and a climbing fiber terminal of the molecular layer (cct, $\left.\boldsymbol{C}_{,} \boldsymbol{C}^{\prime}\right)$. Details at higher magnification are given as insets $\left(\boldsymbol{A}^{\prime}, \boldsymbol{B}^{\prime}, \boldsymbol{C}^{\prime}\right)$. Note that both VGLUT1 and VGLUT2 are present in the mossy fiber terminal $\left(\boldsymbol{A}, \boldsymbol{A}^{\prime}\right)$, while in the parallel fiber terminal only VGLUT1 $\left(\boldsymbol{B}, \boldsymbol{B}^{\prime}\right)$ and in the climbing fiber terminal only VGLUT2 $\left(\boldsymbol{C}, \boldsymbol{C}^{\prime}\right)$ could be detected. Asterisk denotes examples for axon profiles. The small thin arrow indicates an asymmetric synaptic contact. $\boldsymbol{D}, \boldsymbol{D}^{\prime}$, Double immunogold labeling of VGLUT1 (rabbit antiserum, $5 \mathrm{~nm}$ gold particles, forked arrowheads) and VGAT (guinea pig antiserum, $10 \mathrm{~nm}$ gold particles, arrowheads) in a mossy fiber terminal (cmt) of the granular cell layer. A detail a thigher magnification is given as an inset $\left(\boldsymbol{D}^{\prime}\right) . \boldsymbol{E}, \boldsymbol{F}$, Cerebellar basket cell terminals (cbt) were subjected to a double immunogold labeling with VGAT (guinea pig antiserum, $10 \mathrm{~nm}$ gold particles, arrowheads) and rabbit antisera against either VGLUT1 or VGLUT2 ( $5 \mathrm{~nm}$ gold particles, forked arrowheads). Note that basket cell terminals have beside VGAT only VGLUT2. Asterisks denote examples for axon profiles; thick open arrows indicate symmetric contacts; $p c$, Purkinje cell body; pd, Purkinje cell dendrite; sp, dendritic spine. Scale bars: $A-D$ (in $A), E, F$ (in $F), 200 \mathrm{~nm} ; A^{\prime}-D^{\prime}, 100 \mathrm{~nm}$.

When cerebellar mossy fiber terminals were double labeled for VGLUT1 and VGLUT2, VGLUT2 was detected in addition to the expected VGLUT1 (Fig. 5A). In contrast, the molecular layer parallel fiber terminals exclusively displayed VGLUT1- labeling (Fig. 5B), while climbing fiber terminals were immunopositive for VGLUT2 only (Fig. 5C), confirming earlier observations (Fremeau et al., 2004). In cerebellar mossy fiber terminals, in addition to VGLUT also VGAT (Fig. 5D), GAD65, and GAD67 expression was observed (data not shown). Thus, hippocampal and cerebellar mossy fiber terminals seem to be similarly equipped with neurotransmitter transporters and hence may potentially corelease GABA and glutamate. In contrast, the other two types of glutamatergic cerebellar terminals arising from parallel and climbing fibers contained neither VGAT nor one of the GAD isoforms (data not shown).

We also analyzed cerebellar basket cell terminals representing a GABAergic terminal type of the cerebellar cortex. As expected, VGAT (Fig. 5E, F) and GAD65 or GAD67 (data not shown) colocalized in these GABAergic terminals. Surprisingly, we also detected VGLUT2 (Fig. $5 F$ ) but not VGLUT1 (Fig. 5E) besides VGAT in these terminals.

Finally, we quantified the labeling results for VGLUT1, VGLUT2, and VGAT in hippocampal and cerebellar terminals from two animals. For the quantification of VGAT in hippocampal mossy fiber terminals (HMF), only terminals with considerable labeling, containing $>10$ gold particles, were included. These represented $\sim 40 \%$ of all terminals for each animal (70-80 per animal). Although absolute labeling intensities varied between animals, the ratio between VGLUT1 and VGAT labeling was comparable (Fig. 6A). Together with the single-cell mRNA analysis, these findings validate the specificity of the VGAT labeling and confirm the presence of VGAT in large mossy fiber terminals of the hippocampus.

The quantification given in Figure $6 B$ confirmed the specificity of our immunogold labeling.

An exclusive occurrence of VGLUT1 and VGLUT2 is seen in cerebellar parallel fiber terminals (CPFs) and cerebellar climbing fiber terminals (CCFs), respectively. VGLUT2 coexists with VGAT in the basket cell terminals. Comparable to the hippocampal mossy fibers, cerebellar mossy fibers are equipped with all three transporters.

\section{Discussion}

The classical view of individual neurons releasing one type of neurotransmitter has been challenged during recent years. Indeed, the coexistence and corelease of several combinations of classical transmitters with peptides or ATP has been shown to occur in different neuronal 

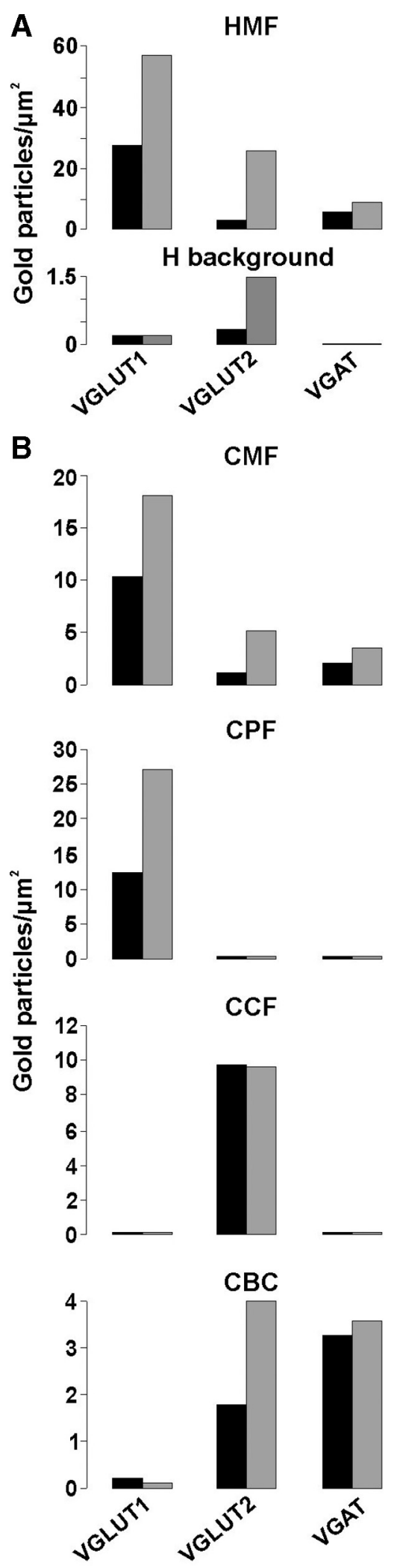

Figure 6. Quantification of the number of gold particles staining for VGLUT1, VGLUT2, and VGAT in glutamatergic and GABAergic terminals of the hippocampal CA3 area and the cerebellum. $\boldsymbol{A}$, The amounts of gold particles indicating either VGLUT1 (rabbit antiserum) or VGAT (guinea pig antiserum, 131004, Synaptic System) in HMFs were quantified in two to three sections of the $C A 3$ area each from separately embedded tissue of two different animals. Single immunogold labeling was performed using the antibodies indicated and immunoreactivity was identified by secondary antibodies coupled to $10 \mathrm{~nm}$ gold particles. For VGAT, only HMFs yielding a labeling with at least 10 gold particles were included, which represent $\sim 40 \%$ of all systems (for review, see Gutiérrez, 2008a). The discovery of VGLUT3 as a cotransmitter transporter supported the idea that glutamate may also be stored in putatively nonglutamatergic neurons (Gras et al., 2008; Seal et al., 2008).

Using different approaches, namely immunoisolation experiments, vesicular transmitter uptake studies, single-cell PCR, and postembedding immunogold electron microscopy, we provide evidence that a coexistence of VGLUT with other neurotransmitter transporters is not restricted to VGLUT3 but also applies to VGLUT1, VGLUT2, and VGAT depending on the neuron type investigated. The simultaneous occurrence of vesicular transport systems associated with both the major excitatory and inhibitory neurotransmitters, glutamate and GABA, provides direct morphological evidence for their corelease under in vivo conditions (Fig. 6).

\section{Vesicular coexistence of transmitter transporters}

Immunoisolation using antibodies against vesicular transmitter transporters yields transmitter-specific vesicular subpopulations still able to concentrate the respective transmitters (Takamori et al., 2000, 2006; Herzog et al., 2006). Using this approach, we found that VGLUT2 occurs on VGAT immunoisolates and thus may also affect the efficiency of GABA uptake. Indeed VGLUT activity contributes to the vesicular loading of GABA mediated by VGAT and monoamines mediated by VMAT2, comparable to the enhancement of VAChT-dependent acetylcholine uptake by VGLUT3 (Gras et al., 2008). Based on the electron microscopic analysis of cerebellar basket cells, the enhancement of GABA uptake is more likely to be mediated by either VGLUT2 or VGLUT3, depending on the type of GABAergic neuron. With monoamine uptake, it is not yet clear whether VGLUT1/2 or alternatively VGLUT3 is responsible for the improvement in transport efficiency. Very recently VGLUT2 has been shown to be required for proper dopamine function (Birgner et al., 2010), which may also include an increase of dopamine storage at the vesicular level.

Regardless of the VGLUT isoforms involved, the negatively charged glutamate directly alters the electrochemical gradient over the vesicle membrane mainly by increasing $\Delta \mathrm{pH}$. The influx of negative charges is neutralized by protons provided by the proton pump. This increase in $\Delta \mathrm{pH}$ improves monoamine as well as GABA uptake, both relying on $\Delta \mathrm{pH}$. It is not clear, however, whether the presence of VGLUT on monoamine- or GABA-storing vesicles necessarily means that glutamate is indeed stored in these vesicles. Interestingly, VGLUT also transports chloride (Schenck et al., 2009). Increasing the vesicular chloride content affects $\Delta \mathrm{pH}$ in the same way as glutamate uptake.

$\leftarrow$

HMFs analyzed per animal (70-80 in total). For each antibody, 30 terminals were analyzed. HMFs with an average area of $4.3 \pm 0.3 \mu \mathrm{m}^{2}$ were considered and values calculated as gold particles per $1 \mu \mathrm{m}^{2}$. Nonspecific labeling (H background) obtained from dendritic cytoplasm and nuclei over an area of $20 \mu \mathrm{m}^{2}$ for each section and antibody is given below and was subtracted. Note that background labeling was $<10 \%$ under each condition. $\boldsymbol{B}$, In a similar experimental design, the immunogold staining in the various terminals of cerebellum was quantified. For each animal, 40 [cerebellar mossy fibers (CMFs), cerebellar parallel fiber terminals (CPFs)] or 20 [cerebellar climbing fiber terminals (CCFs) or cerebellar basket cell terminals (CBCS)] were analyzed. The average areas for the terminals considered were the following: CMFs, $9.92 \pm 0.68 \mu \mathrm{m}^{2}$; CPFs, $0.49 \pm 0.06 \mu \mathrm{m}^{2}$; CCFs, $0.93 \pm 0.12 \mu \mathrm{m}^{2}$; CBFs, $1.27 \pm 0.075 \mu \mathrm{m}^{2}$. Values are given as gold particles per $1 \mu \mathrm{m}^{2}$. There was no correction for background labeling. Note that CPFs were exclusively immunopositive for VGLUT1, whileCCFs have only VGLUT2. CBFs have both VGAT and VGLUT2, while mossy fiber terminals contain all three transporters. 
In addition to VGLUT1-3, VGAT, and VMAT1/2, other small solute carriers (SLCs) have been identified during the last years. Detailed analysis revealed that a growing number of these transporters also occurs on SVs, like the vesicular excitatory amino acid transporter VEAT or sialin (Morin et al., 2004; Wreden et al., 2005; Yarovaya et al., 2005; Miyaji et al., 2008), and Rxt1/NTT4, which is responsible for vesicular accumulation of nonpolar amino acids (Masson et al., 1999; Takamori et al., 2006; Parra et al., 2008). The additional presence of these transporters increases the complexity of regulatory processes at the presynaptic level with impact on postsynaptic activity. Specific sets of various SLC transporters may thus help to shape the transmitter profile of individual synapses and vesicles.

\section{Synaptic coexistence of VGLUT and VGAT}

The expression of various transmitter transporters on either different or the same vesicles within the same terminal requires regulatory processes to control vesicle recruitment under physiological conditions (Gutiérrez, 2008a,b). Apparently, VGLUT1 and VGLUT2 either occur on different SVs segregated to different release sites (Fremeau et al., 2004) or colocalize on the same SVs (Wojcik et al., 2004; Herzog et al., 2006; this investigation). While a coexpression of VGLUT1 and VGLUT2 will necessarily result in the release of glutamate only, the situation is clearly different when confronted with a synaptic coexistence of VGLUT and VGAT.

\section{VGLUT in GABAergic neurons}

The ability of GABAergic cells to release glutamate was proven by recording glutamate-mediated currents from VGLUT1transfected GABAergic neurons establishing autaptic synapses (Takamori et al., 2000). During development of the auditory system GABAergic neurons of the lateral superior olive also release glutamate. This corelease of glutamate activates NMDAreceptors mediating $\mathrm{Ca}^{2+}$ entry in the postsynaptic neuron and is necessary to enforce synaptic strength (Gillespie et al., 2005). Thus, in GABAergic neurons VGLUT may serve different tasks. First, it enables GABAergic neurons to release glutamate, thereby modulating postsynaptic responses and synaptic strength, e.g., during development. Second, VGLUT may improve vesicular GABA loading and storage (see above) and thereby modulate GABAergic transmission. This may partly explain why VGLUT3 knock-out animals develop nonconvulsive seizures (Seal et al., 2008). To improve vesicular GABA storage, VGLUT may also transport chloride instead of glutamate (Schenck et al., 2009), thus avoiding a corelease of excitatory and inhibitory neurotransmitters.

\section{VGAT in glutamatergic neurons}

In contrast to VGLUT, possibly improving GABA uptake in GABAergic terminals, the functional relevance of VGAT expression in distinct types of glutamatergic terminals appears less obvious.

All CA3 mossy fiber terminals investigated in this study contained VGLUT1 and VGLUT2 immunoreactivity, and thus qualify to be classified as glutamatergic terminals. Using immunogold detection, Bergersen et al. (2003) showed that hippocampal mossy fiber terminals contained both glutamate and GABA. In $\sim 40 \%$ of hippocampal mossy fiber terminals, we also detected considerable amounts of VGAT immunoreactivity. Similarly, in cerebellar mossy fiber terminals, VGAT was colocalized with VGLUT1 and VGLUT2, though here all mossy fiber terminals expressed the vesicular GABA transporter. In addition to single- cell PCR data demonstrating the presence of VGAT mRNA in hippocampal granule cells (Gómez-Lira et al., 2005; this investigation), we show here the coexistence of VGAT and VGLUT in mossy fiber terminals at the electron microscopic level using a range of VGLUT and VGAT antibodies. Comparable to the auditory system (Gillespie et al., 2005), GABA and glutamate may be released simultaneously from developing hippocampal mossy fiber terminals (Walker et al., 2001; Gutierrez et al., 2003; Kasyanov et al., 2004; Safiulina et al., 2006). The coexpression of GABA and glutamate transporters in glutamatergic terminals argues in favor of a corelease of glutamate and GABA, possibly involving different scenarios. In the developing brain, the concerted action of coreleased neurotransmitters appears to be fundamentally required to shape neuronal circuits (Gutiérrez, 2005, 2008b). Most neurons express neurotransmitter autoreceptors in their terminals as well as in the preterminal regions of their axons (Ruiz et al., 2003). Different release sites at central synapses exist in $\alpha$-motoneurons, which despite exciting muscles and other motoneurons by acetylcholine, also release glutamate, thereby mediating an excitatory feedback to the inhibitory Renshaw cells (Nishimaru et al., 2005). Also, supramammillary nucleus neurons synapsing onto dendrites of dentate gyrus granule cells form symmetric as well as asymmetric contacts. Thus, the presence of VGLUT2 and VGAT in these terminals indicates glutamate and GABA release at different release sites (Boulland et al., 2009), although corresponding electrophysiological data are so far not available.

The stimulation protocols generally used to activate mossy fibers cannot definitely exclude that a synchronous release from adjacent GABAergic terminals may underlie the observed effects (Uchigashima et al., 2007). However, GABA is apparently stored in a subset of glutamatergic terminals (hippocampal and cerebellar mossy fiber terminals) but absent in others (cerebellar parallel and climbing fiber terminals), thus supporting a specificity seemingly characteristic of large complex glutamatergic terminals enriched with synaptic vesicles. The coexistence of GABA in these terminals provides the mossy fibers with a putative mechanism to autoregulate synaptic release via activation of presynaptic GABA $_{\mathrm{A}}$ (Ruiz et al., 2003; Treviño and Gutiérrez, 2005; Alle and Geiger, 2007) and/or GABA $_{B}$ receptors (Chandler et al., 2003). Based on these considerations, it is tempting to speculate that GABA release may modulate glutamate release via presynaptic $\mathrm{GABA}_{\mathrm{A}}$ and/or $\mathrm{GABA}_{\mathrm{B}}$ receptors. Such a modulation of glutamate release by GABA would indeed not have been detected under conditions of blocking all types of postsynaptic glutamate receptors by antagonists.

Assuming that GABA may exert its effects presynaptically by modulating glutamate release, this may be more important in the developing than in adult hippocampus. Following excessive or pathological stimulation, GABAergic transmission increases to counteract harmful excitation, which also involves presynaptic receptors (Chandler et al., 2003; Treviño and Gutiérrez, 2005). Thus, adult CA3 mossy fibers still exhibiting profound GABAergic properties may overcome overexcitations, thereby preventing the development of epileptiform activity. Whether GABA is released from the same or different vesicles is still not clear. Regarding the additional presence of VGLUT2 in mossy fiber terminals, this transporter may function by promoting GABA loading in VGAT vesicles, thereby increasing the GABA response.

These results indicate that both a synaptic and, presumably, a vesicular coexistence of VGLUT and VGAT in varying combinations do occur in distinct glutamatergic and GABAergic terminals. The functions of these transporters, seemingly 
contrary at first glance, may be important to differentially fine-tune and shape synaptic efficiency during the development of neuronal networks as well as during adaptive processes in the adult brain.

\section{References}

Alle H, Geiger JRP (2007) GABAergic spill-over transmission onto hippocampal mossy fiber boutons. J Neurosci 27:942-950.

Becher A, Drenckhahn A, Pahner I, Margittai M, Jahn R, Ahnert-Hilger G (1999) The synaptophysin-synaptobrevin complex —a hallmark of vesicle maturation. J Neurosci 19:1922-1931.

Bergersen L, Ruiz A, Bjaalie JG, Kullmann DM, Gundersen V (2003) GABA and GABAA receptors at hippocampal mossy fibre synapses. Eur J Neurosci 18:931-941.

Birgner C, Nordenankar K, Lundblad M, Mendez JA, Smith C, le Grevès M, Galter D, Olson L, Fredriksson A, Trudeau L-E, Kullander K, WallénMackenzie A (2010): Vglut2 in dopamine neurons is required for psychostimulant-induced behavioral activation. Proc Natl Acad Sci U S A 107:389-394.

Boulland JL, Jenstad M, Boekel AJ, Wouterlood FG, Edwards RH, StormMathisen J, Chaudhry FA (2009) Vesicular glutamate and GABA transporters sort to distinct sets of vesicles in a population of presynaptic terminals. Cereb Cortex 19:241-248.

Brunk I, Blex C, Rachakonda S, Höltje M, Winter S, Pahner I, Walther DJ, Ahnert-Hilger G (2006) The first lumenal domain of the vesicular monoamine transporter 2 mediates G-protein-dependent regulation of transmitter uptake. J Biol Chem 281:33373-33385.

Chandler KE, Princivalle AP, Fabian-Fine R, Bowery NG, Kullmann DM, Walker MC (2003) Plasticity of $\mathrm{GABA}_{\mathrm{B}}$ receptor-mediated heterosynaptic interactions at mossy fibers after status epilepticus. J Neurosci 23:11382-11391.

Chaudhry FA, Reimer RJ, Bellocchio EE, Danbolt NC, Osen KK, Edwards RH, Storm-Mathisen J (1998) The vesicular GABA transporter, VGAT, localizes to synaptic vesicles in sets of glycinergic as well as GABAergic neurons. J Neurosci 18:9733-9750.

Chen W, Mahadomrongkul V, Berger UV, Bassan M, DeSilva T, Tanaka K, Irwin N, Aoki C, Rosenberg PA (2004) The glutamate transporter GLT1a is expressed in excitatory axon terminals of mature hippocampal neurons. J Neurosci 24:1136-1148.

Edwards RH (2007) The neurotransmitter cycle and quantal size. Neuron 55:835-858.

Fremeau RT Jr, Kam K, Qureshi T, Johnson J, Copenhagen DR, StormMathisen J, Chaudhry FA, Nicoll RA, Edwards RH (2004) Vesicular glutamate transporters 1 and 2 target to functionally distinct synaptic release sites. Science 304:1815-1819.

Gillespie DC, Kim G, Kandler K (2005) Inhibitory synapses in the developing auditory system are glutamatergic. Nat Neurosci 8:332-338.

Gómez-Lira G, Lamas M, Romo-Parra H, Gutiérrez R (2005) Programmed and induced phenotype of the hippocampal granule cells. J Neurosci 25:6939-6946.

Gras C, Amilhon B, Lepicard EM, Poirel O, Vinatier J, Herbin M, Dumas S, Tzavara ET, Wade MR, Nomikos GG, Hanoun N, Saurini F, Kemel M-L, Gasnier B, Giros B, El Mestikawy S (2008) The vesicular glutamate transporter VGLUT3 synergizes striatal acetylcholine tone. Nat Neurosci 11:292-300.

Gutiérrez R (2005) The dual glutamatergic-GABAergic phenotype of the hippocampal granule cells. Trends Neurosci 28:297-303.

Gutiérrez R (2008a) Co-release of classical neurotransmitters. In: Coexistence and co-release of classical neurotransmitters. Ex uno plures (Gutiérrez R, Ed), pp 15-22. Berlin: Springer.

Gutiérrez R (2008b) The dual glutamatergic/GABAergic phenotype of the hippocampal granule cells. In: Co-existence and co-release of classical neurotransmitters. Ex uno plures (Gutiérrez R, ed). pp 181-202. Berlin: Springer.

Gutiérrez R, Heinemann U (2001) Kindling induces transient fast inhibition in the dentate gyrus-CA3 projection. Eur J Neurosci 13:1371-1379.

Gutiérrez R, Romo-Parra H, Maqueda J, Vivar C, Ramírez M, Morales MA, Lamas M (2003) Plasticity of the GABAergic phenotype of the "glutamatergic" granule cells of the rat dentate gyrus. J Neurosci 23:5594-5598.

Herzog E, Takamori S, Jahn R, Brose N, Wojcik SM (2006) Synaptic and vesicular co-localization of the glutamate transporters VGLUT1 and VGLUT2 in the mouse hippocampus. J Neurochem 99:1011-1018.
Kao YH, Lassová L, Bar-Yehuda T, Edwards RH, Sterling P, Vardi N (2004) Evidence that certain bipolar cells use both glutamate and GABA. J Comp Neurol 478:207-218.

Kasyanov AM, Safiulina VF, Voronin LL, Cherubini E (2004) GABAmediated giant depolarizing potentials as coincidence detectors for enhancing synaptic efficacy in the developing hippocampus. Proc Natl Acad Sci U S A 101:3967-3972.

Martens H, Weston MC, Boulland JL, Grønborg M, Grosche J, Kacza J, Hoffmann A, Matteoli M, Takamori S, Harkany T, Chaudhry FA, Rosenmund C, Erck C, Jahn R, Härtig W (2008) Unique luminal localization of VGAT-C terminus allows for selective labeling of active cortical synapses. J Neurosci 28:13125-13131.

Masson J, Riad M, Chaudhry F, Darmon M, Aïdouni Z, Conrath M, Giros B, Hamon M, Storm-Mathisen J, Descarries L, El Mestikawy S (1999) Unexpected localization of the $\mathrm{Na}+/ \mathrm{Cl}$-dependent-like orphan transporter, rxt1, on synaptic vesicles in the rat central nervous system. Eur J Neurosci 11:1349-1361.

McIntire SL, Reimer RJ, Schuske K, Edwards RH, Jorgensen EM (1997) Identification and characterization of the vesicular GABA transporter. Nature 389:870-876

Miyaji T, Echigo N, Hiasa M, Senoh S, Omote H, Moriyama Y (2008) Identification of a vesicular aspartate transporter. Proc Natl Acad Sci U S A 105:11720-11724.

Morin P, Sagné C, Gasnier B (2004) Functional characterization of wild type and mutant human sialin. EMBO J 23:4560-4570.

Nishimaru H, Restrepo CE, Ryge J, Yanagawa Y, Kiehn O (2005) Mammalian motoneurons co-release glutamate and acetylcholine at central synapses. Proc Natl Acad Sci U S A 102:5245-5249.

Ottem EN, Godwin JG, Krishnan S, Petersen SL (2004) Dual-phenotype GABA/glutamate neurons in adult preoptic area: sexual dimorphism and function. J Neurosci 24:8097-8105.

Pahner I, Höltje M, Winter S, Takamori S, Bellocchio EE, Spicher K, Laake P, Nürnberg B, Ottersen OP, Ahnert-Hilger G (2003) Functinal G-protein heterotrimers are associated with vesicles of putative glutamatergic terminals: implications for regulation of transmitter uptake. Mol Cell Neurosci 23:398-413.

Parra LA, Baust T, El Mestikawy S, Quiroz M, Hoffman B, Haflett JM, Yao JK, Torres GE (2008) The orphan transporter Rxt1/NTT4 (SLC6A17) functions as a synaptic vesicle amino acid transporter selective for proline, glycine, leucine and alanine. Mol Pharmacol 74:1521-1532.

Roseth S, Fykse EM, Fonnum F (1998) Uptake of L-glutamate into synaptic vesicles: competitive inhibition by dyes with biphenyl and amino- and sulphonic acid-substituted naphthyl groups. Biochem Pharmacol 56: 1243-1249.

Ruiz A, Fabian-Fine R, Scott R, Walker MC, Rusakov DA, Kullmann DM (2003) GABAA receptors at hippocampal mossy fibers. Neuron 39:961-973.

Safiulina VF, Fattorini G, Conti F, Cherubini E (2006) GABAergic signaling at mossy fiber synapses in neonatal rat hippocampus. J Neurosci 26:597-608.

Schenck S, Wojcik SM, Brose N, Takamori S (2009) A chloride conductance in VGLUT1 underlies maximal glutamate loading into synaptic vesicles. Nat Neurosci 12:156-162.

Seal RP, Akil O, Yi E, Weber CM, Grant L, Yoo J, Clause A, Kandler K, Noebels JL, Glowatzki E, Lustig LR, Edwards RH (2008) Sensorineural deafness and seizures in mice lacking the vesicular glutamate transporter 3. Neuron 57:263-275.

Sloviter RS, Dichter MA, Rachinsky TL, Dean E, Goodman JH, Sollas AL, Martin DL (1996) Basal expression and induction of glutamate decarboxylase and GABA in excitatory granule cells of the rat and monkey hippocampal dentate gyrus. J Comp Neurol 373:593-618.

Sperk G, Schwarzer C, Heilman J, Furtinger S, Reimer RJ, Edwards RH, Nelson N (2003) Expression of plasma membrane GABA transporters but not of the vesicular GABA transporter in dentate granule cells after kainic acid seizures. Hippocampus 13:806-815.

Takamori S, Riedel D, Jahn R (2000) Immunoisolation of GABA-specific synaptic vesicles defines a functional distinct subset of synaptic vesicles. J Neurosci 20:4904-4911.

Takamori S, Holt M, Stenius K, Lemke EA, Grønborg M, Riedel D, Urlaub H, Schenck S, Brügger B, Ringler P, Müller SA, Rammner B, Gräter F, Hub 
JS, De Groot BL, Mieskes G, Moriyama Y, Klingauf J, Grubmüller H, Heuser J, et al. (2006) Molecular anatomy of a trafficking organelle. Cell 127:831-846.

Treviño M, Gutiérrez R (2005) The GABAergic projection of the dentate gyrus to hippocampal area CA3 of the rat: pre- and postsynaptic actions after seizures. J Physiol 567:939-949.

Uchigashima M, Fukaya M, Watanabe M, Kamiya H (2007) Evidence against GABA release from glutamatergic mossy fibre terminals in the developing hippocampus. J Neurosci 27:8088-8100.

Walker MC, Ruiz A, Kullmann DM (2001) Monosynaptic GABAergic signaling from dentate to CA3 with a pharmacological and physiological profile typical of mossy fibre synapses. Neuron 29:703-715.

Winter S, Brunk I, Walther DJ, Höltje M, Jiang M, Peter JU, Takamori S, Jahn $\mathrm{R}$, Birnbaumer L, Ahnert-Hilger G (2005) $\mathrm{G} \alpha_{\mathrm{o} 2}$ regulates vesicular glu- tamate transporter activity by changing its chloride dependence. J Neurosci 25:4672-4680.

Wojcik SM, Rhee JS, Herzog E, Sigler A, Jahn R, Takamori S, Brose N, Rosenmund C (2004) An essential role for vesicular glutamate transporter 1 (VGLUT1) in postnatal development and control of quantal size. Proc Natl Acad Sci U S A 101:7158-7163.

Wreden CC, Wlizla M, Reimer RJ (2005) Varied mechanisms underlie the free sialic acid storage. J Biol Chem 280:1408-1416.

Yarovaya N, Schot R, Fodero L, McMahon M, Mahoney A, Williams R, Verbeek E, de Bondt A, Hampson M, van der Spek P, Stubbs A, Masters CL, Verheijen FW, Mancini GMS, Venter DJ (2005) Sialin, an anion transporter defective in sialic acid storage diseases shows highly variable expression in adult mouse bran, and is developmentally regulated. Neurobiol Dis 19:351-365. 